\title{
A regret-based incremental elicitation for multi-criteria force design
}

\author{
V. Mak-Hau $^{\text {a }}$ (D), T. Cao ${ }^{\text {b }}$, J. Yearwood ${ }^{a}$ \\ a School of Information Technology, Deakin University, Waurn Ponds, Vic 3216, Australia \\ ${ }^{\mathrm{b}}$ Joint and Operations Analysis Division, Defence Science and Technology Group, Australia \\ Email: vicky.mak@deakin.edu.au
}

\begin{abstract}
We aim to develop a framework for defence force design multi-objective decision-making problems where there are multiple decision makers each with a potentially different set of priorities, but only one solution is required in the end in practice.

Benabbou et al. proposed an interactive preference elicitation approach designed for problems with a single decision maker in the Proceedings of the AAAI Conference (2020). In this approach, a linear scalarizing function is used for the multiple objectives. In each iteration, a population of different scalarizing parameter vectors are generated and the associated single-objective optimization problems are solved one by one, each providing a different "optimal" solution. Following that, the search space of the scalarizing parameters is reduced through preference elicitation of pairwise "optimal" solutions obtained from the parameter vector population as each preference produces a cut to the feasible parameter set. The stopping criteria is controlled by the calculation of a regret value. If, in any iteration, out of all solutions found, there is a solution that returns the smallest maximum pairwise regret value and that the value is smaller than a predetermined tolerance, then the solution will be chosen as the final optimal solution.

In this paper, we propose a number of adjustments and modifications to the Benabbou et al. approach required for our intended application - Defence Force Design. We provide an insight on the impact of having multiple decision makers instead of just a single decision maker. It may seem counter-intuitive, but with multiple decision makers, we expect that the search space of the linear scalarizing function parameters converges to a single point much quicker if the decision makers conflict with each other in their preferences. We also propose a hierarchical approach to deal with high dimensional objective space as well as alternative scalarizing function parameter search schemes.
\end{abstract}

Keywords: Multi-objective optimization, Regret model, preference elicitation, multiple decision makers 


\section{INTRODUCTION}

This paper presents a framework for solving defence force design multi-objective optimization problems with multiple decision-makers in the loop. The intended application is complex decision making problems where the decision makers each has a set of potentially conflicting decision criteria.

Multi-objective optimization problems (MOO) are usually solved either with a single optimal solution or a Pareto set in mind. With the former, common approaches are to solve the MOO as a single-objective optimization problem by using some predetermined scalarization function or Lexicographically with a predetermined ordering of the objectives. If more than one solution is desired, then the Pareto front or a near-Pareto front can be exhaustively or partially enumerated. But the question is, even if the optimal Pareto front can be found, how does a Decision Maker (DM) make a decision?

In recent years, preference-based search in multi-objective combinatorial optimization (MOCO) has been proposed in a number of studies, see, e.g., Kaddani et al. [2017], Benabbou and Perny [2016], Benabbou and Perny [2015], and Benabbou and Perny [2018]. Eliciting preferences incrementally based on minimax regret has been proposed in Boutilier et al. [2006], Benabbou and Perny [2015], and Benabbou and Perny [2018].

Recently Benabbou et al. [2020] proposed a minimax regret-based approach for solving MOCO problems that not only incrementally elicits preferences from DMs, but at the same time, these preferences help reduces the search space of the linear scalarizing function parameters but generating a cutting plane from each preference. The paper focused on a single decision maker.

In this paper, we propose to use this approach to tackle the Defence Force Design problems and provide some insights in implementation aspects, in particular, the impact on having multiple decision makers.

\subsection{Multi-objective combinatorial optimization problems}

Consider a set of $m$ decision variables: $x=\left(x_{1}, x_{2}, \ldots, x_{m}\right)$. The domain of each decision variable is a set of discrete values, i.e., $x_{j} \in\left\{1, \ldots, \mathbb{N}_{j}\right\}$ for $j=1, \ldots, m$. Let $\chi$ be the set of all feasible solutions, and $y(x)=\left(y_{1}(x), \ldots, y_{n}(x)\right)$ be the performance vector (i.e., the $n$ objectives). Let $f_{\omega}(x)$ be a linear scalarizing function, for $\omega=\left(\omega_{1}, \ldots, \omega_{n}\right)$ the parameters, with $\omega_{i}>0$ for each $i=1, \ldots, n$. Without loss of generality, we can assume that $\sum_{i=1}^{n} \omega_{i}=1$. We have that

$$
\min _{x \in X} f_{\omega}(y(x))=\sum_{i=1}^{n} \omega_{i} y_{i}(x)
$$

Let $\Omega=\left\{\omega \mid \omega_{i}>0, \forall i=1, \ldots, n, \sum_{i=1}^{n} \omega_{i}=1\right\}$ be the set of feasible parameters. With any given $\omega,(1)$ is a single-objective optimization (SOO) problem and thus one can obtain an optimal or a near-optimal solution by an exact method or a heuristic approach. This paper concerns the search of the parameters $\omega$ in an MOCOP through an incremental preference elicitation process, not the solution method of a SOO problem.

\subsection{A regret-based incremental preference elicitation in MOCOP}

A regret-based incremental preference elicitation (RIPE) is proposed in Benabbou et al. [2020] for solving MOCOPs. The RIPE algorithm will incrementally reduce the feasible set of the parameters of the linear scalarization function $(\omega)$ by asking a decision-maker to compare pairs of "optimal solutions", e.g., $\tilde{x}^{1}$ and $\tilde{x}^{2}$ obtained from distinct $\omega^{1}, \omega^{2} \in \Omega$ respectively, and provide a preference. If the decision-maker decides that $\tilde{x}^{1}$ is better than $\tilde{x}^{2}$, for example, this gives us:

$$
f_{\omega}\left(y\left(\tilde{x}^{1}\right)\right) \leq f_{\omega}\left(y\left(\tilde{x}^{2}\right)\right)
$$

Notice that in this inequality, $\omega$ are variables and $y\left(\tilde{x}^{1}\right)$ are known, and therefore the inequality will provide us with a linear constraint on $\omega$, that is, a cutting plane on the polyhedron $\Omega$.

Then, a pairwise regret value for $\tilde{x}^{1}$ and $\tilde{x}^{2}$ is calculated as:

$$
\max _{\omega \in \Omega_{t}}\left\{f_{\omega}\left(y\left(\tilde{x}^{1}\right)\right)-f_{\omega}\left(y\left(\tilde{x}^{2}\right)\right)\right\}
$$

where $\Omega_{t}$ is the set of parameter vectors $\omega$ used in iteration $t$. (The size of $\left|\Omega_{t}\right|$ is predetermined by the user or a DM). 
Now, for each optimal solution $\tilde{x}$ obtained in an iteration, we find the maximum regret out of the comparisons with all other optimal solutions $\tilde{x}^{\prime} \neq \tilde{x}$, and then the solution $\tilde{x}^{*}$ with the minimum maximum regret. If the value of the this mini-max regret is smaller than a pre-determined tolerance, the solution(s) that provide the mini-max regret value will be reported as an output, otherwise, the RIPE procedure will continue.

\section{THE DEFENCE FORCE DESIGN PROBLEM}

The Force Structure Plan (FSP) is the outcome of the of the force options that are explored and tested through the Australian Capability Context Scenarios (ACCS) in the Defence Capability Assessment Program. Department of Defence describes the ACCS as possible circumstances under which the future joint force might be employed. The complexity of the scenario-based problems and the number of dimensions across whole-offorce makes it almost impossible to apply detailed traditional modelling approach such as combat simulation. Consequently, a decision support model, that traded-off fidelity for feasibility and relied on structured elicitation of multiple subject matter experts (SMEs) was employed (see Peacock et al. [2019]). Force Design (FD) is ultimately about selecting a portfolio of thousands of strategic investment projects in future Australian Defence Force (ADF) capabilities. The challenge is to optimise the combination of invested projects that give government the overall capability it requires to achieve multiple effects (objectives) across a range of scenarios, whilst achieving the budget constraints. To address the complex and uncertain nature of force design, Defence Science and Technology Group are collaborating with Deakin University to develop the novel methodology for solving multiobjective optimization problems with complex large scale whole of force. This paper aims to extend some earlier approaches successfully used for evaluating and optimising small scale defence problems such as the Land Combat Vehicle (LCV) system specifications and configurations for the Australian Army (see Nguyen and Cao [2019]), to more complex problems with higher dimensions that are relevant to whole of force design.

\subsection{Past solution space search algorithms}

Traditionally, the optimal defence system design solution was found by creating a single aggregate objective function and using a weighted sum approach. This method may have some drawbacks, since it is often very difficult to numerically quantify how important the objectives (i.e. its weighting) are relative to each other, resulting in designs which do not really best meet the DM's goals (see Nguyen and Cao [2017]). Multi-objective optimisation attempts to solve this problem by using the concept of Pareto optimality to find non-dominated solutions (see Nguyen and Cao [2019]). A solution is said to be non-dominated when there is no other solution in the space which is better with regards to all decision variables. It is not always possible to find Pareto-optimal points, Multi-Objectives Evolutionary Algorithm (MOEA) was developed to approximate the Pareto optimal set (see Hadka and Reed [2012]) by evolving a population of solutions to find multiple non-dominated points as close to the Pareto-optimal front as possible, with a wide trade-off among objectives. It is heuristic based, so may not be guaranteed in finding Pareto-optimal points. But it has essential operators to constantly improve the evolving non-dominated points similar to the way most natural and artificial evolving systems continuously improve their solutions. A MOEA algorithm developed by Hadka [2014] with NSGA-II algorithm option Deb et al. [2002] had been successfully applied in our previous work (see Nguyen and Cao [2019]) for searching the 'best' configuration of a small scale defence system (Land Combat Vehicle System) with four objectives: survivability, lethality, mobility and knowledge. However, that approach is not feasible for large scale problem such as whole of force design due to complexity and high dimension of objectives which generate very large search space. Consequently, our aim in this paper is to develop an interactive approach with decision makers to reduce search space for finding Pareto-optimal points in whole of force design.

\section{A FRAMEWORK FOR IMPLEMENTING RIPE IN DEFENCE FORCE DESIGN}

In this section, we discuss how the RIPE framework can be implemented to the Defence Force Design MCDM described earlier as well as the adjustments and modifications required.

The initial steps are as follows. Assume that a heuristic approach is used to search the space of $\Omega$. Let $\Omega_{0}, \Omega_{1}, \Omega_{2}, \ldots$ be the sequence of reduced search space of $\Omega$ obtained throughout the RIPE iterations. The method begins with the polyhedron $\Omega_{0}=\left\{\omega \mid\left(w_{1}, \ldots, w_{n}\right) \in\{0,1\}^{n}, \forall i=1, \ldots, n, \sum_{i=1}^{n} \omega_{i}=1\right\}$. This gives us $n$ extreme points of the polyhedron. Each extreme point represents one of the objective functions which has a weight of 1 , and all other objective function have a weight of 0 .

If a Genetic Algorithm-type approach is used to search the space of $\Omega$, and if the population size $P$ is chosen to be larger than $n$, then $P-n$ extra points are required, which can be chosen by randomly selecting some 
interior points in $\Omega$, to give $\Omega_{0}$.

Notice one can also have the initial set of parameter vectors randomly chosen in $\Omega_{0}=\left\{\omega \mid\left(w_{1}, \ldots, w_{n}\right) \in\right.$ $\left.[0,1]^{n}, \forall i=1, \ldots, n, \sum_{i=1}^{n} \omega_{i}=1\right\}$.

Let $\Theta_{t}$ be the set of available preference information in Iteration $t$ of the RIPE algorithm. We have that $\Theta_{0}=\emptyset$, and we find $\Theta_{1}$ by performing the following steps. For each of parameter set in the initial population (of size $P$ ), that is, for each $\omega^{0, j} \in \Omega_{0}$, where $j=1, \ldots, P$, an optimal solution $x_{\omega^{0, j}}^{*} \in \chi$ to (1) is obtained (e.g., by a heuristic method). This gives us the set $\chi^{0}$, the set of optimal solutions obtained in Iteration 0 .

We then ask the decision-makers to evaluate pairs of solutions in $\chi^{0}$. These preferences give us the set $\Theta_{1}$. With these preferences, we are able to update $\Omega$ by adding new cuts in the form of (2).

We use $(a, b)$ to denote that solution $x^{a}$ is more preferable than solution $x^{b}$, after the DM makes his/her choice. We have that:

$$
\Omega_{1}=\left\{\omega: \forall(a, b) \in \Theta_{1}, f_{\omega}\left(y\left(x^{a}\right)\right) \leq f_{\omega}\left(y\left(x^{b}\right)\right)\right\}
$$

Now, we calculate a Pairwise Regret (PR) for a pair of solutions $x, x^{\prime}$ in $\chi^{0}$, finding the parameters $\omega \in \Omega_{1}$ that makes solution $x$ least preferred when compared to $x^{\prime}$ (as we assume that the MOCOP is a minimization problem for all objectives):

$$
P R\left(x, x^{\prime}, \Omega_{1}\right)=\max _{\omega \in \Omega_{1}}\left\{f_{\omega}(y(x))-f_{\omega}\left(y\left(x^{\prime}\right)\right)\right\}
$$

Out of all PRs for solution $x \in \chi^{0}$, we find the Maximum Regret (MR):

$$
M R\left(x, \chi_{0}, \Omega_{1}\right)=\max _{x^{\prime} \in \chi_{0}} P R\left(x, x^{\prime}, \Omega_{1}\right)
$$

Finally, we calculate the Minimax Regret (MMR) out of all the MRs calculated from all $x \in \chi^{0}$.

$$
\operatorname{MMR}\left(\chi_{0}, \Omega_{1}\right)=\min _{x \in \chi_{0}} \operatorname{MR}\left(x, \chi_{0}, \Omega_{1}\right)
$$

The solution(s) in $\arg \min _{x \in \chi_{0}} M R\left(x, \chi, \Omega_{1}\right)$ is the "preferred" solution(s) in $\chi^{0}$ according to the Minimax Regret.

Then, we find new parameter sets $\omega$ in $\Omega_{1}$ and repeat the procedure. The procedure can be terminated when the Minimax Regret obtained at some iteration is smaller than a predetermined tolerance $\delta$. Suppose that this is achieved in Iteration $t$, then the optimal solution(s) in $\arg \min _{x \in \chi_{t}} M R\left(x, \chi_{t}, \Omega_{t+1}\right)$ is/are the final solution(s) to be recommended to the decision-maker.

Figure 1 provides a flow chat for the RIPE algorithm.

\subsection{Multiple Decision Makers}

If $\chi^{t}$ is large and there are multiple DMs, the set of solution pairs can be distributed to different DMs so as to speed up the comparison process. There is, however, a benefit in asking multiple DMs to assess the same pairs of solutions.

When a decision-maker prefers solution $\tilde{x}^{1}$ over $\tilde{x}^{2}$, we obtain the half-space defined by (2). If, however, another decision-maker prefers $\tilde{x}^{2}$ over $\tilde{x}^{1}$, then we have a region defined by the intersection of the two halfspace, which is a hyperplane:

$$
f_{\omega}\left(y\left(\tilde{x}^{1}\right)\right)=f_{\omega}\left(y\left(\tilde{x}^{2}\right)\right)
$$

Clearly, the larger the population $P$ is (i.e., the more "optimal" solutions obtained in each iteration $\chi^{t}$ ), the more decision makers are involved, and more importantly, if the decision makers have very different criteria in mind, the more likely $\Omega_{t}$ can be significantly reduced.

In fact, as soon as we have $n$ sets of conflicting preferences, and the resulting hyperplanes are linearly independent, $\Omega$ will converge to a unique solution.

An interesting computational experiment would be to see which of the following occurs first: a unique set of $\omega$ is found, or the mini-max regret falls below the predetermined tolerance value. As they both serve as a stopping criteria, it would be interesting to see, in practice, which one occurs first for the intended application. 


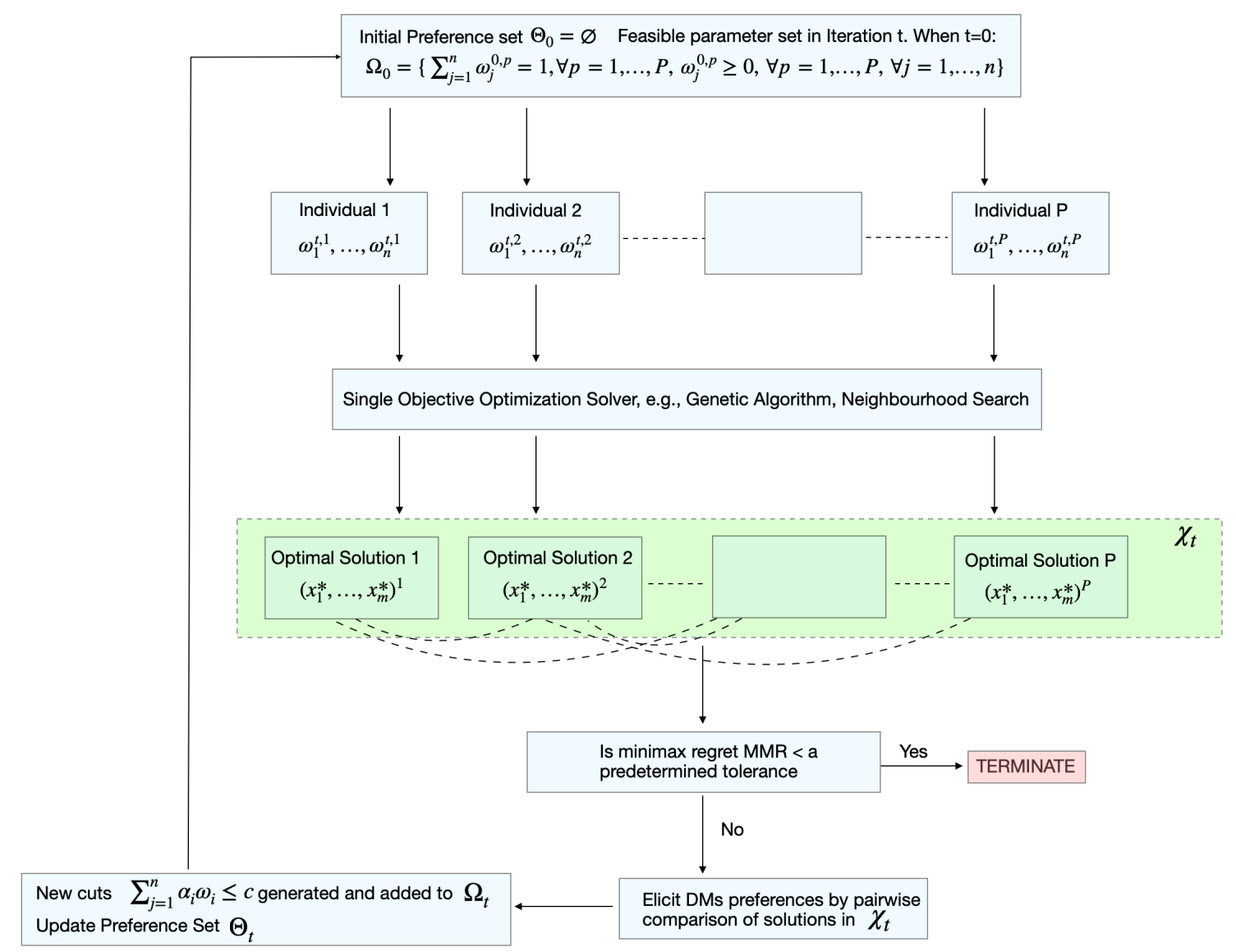

Figure 1. A flow chat for the RIPE algorithm.

\subsection{Updating $\Omega_{t}$}

There are a number of approaches for updating $\Omega_{t}$.

1. In Benabbou et al. [2020], given two parameter vectors used in iteration $t, \omega^{t, 1}, \omega^{t, 2} \in \Omega^{t}$, a new parameter vector in iteration $t+1$ can be obtained by $\omega=\lambda \omega^{1}+(1-\lambda) \omega^{2}$, for $\lambda \in(0,1)$. The calculation is fast and straight forward, however the points $\omega^{1}$ and $\omega^{2}$ must be in the intersection of the feasible set $\Omega^{t}$ and the new cuts (2) generated.

2. In any iteration $t$, with $\Omega_{t}$, one can obtain a set of parameter vectors $\omega^{t, 1}, \ldots, \omega^{t, P}$ by randomly generating $P$ points in $\Omega_{t}$ using, for example, the method described in Meister and Clauss [2020].

3. In iteration $t+1$, obtain $P$ new parameter vectors from the $P$ parameter vectors obtained in iteration $t$ by performing a neighbourhood search. A new point $\omega^{t+1, j}, j=1, \ldots, P$ can be randomly selected from the intersection of a ball of diameter $r$ centered at Point $\omega^{t, j}$ and the new cuts generated in iteration $t$.

\subsection{Repetitions and mini-mini-max regret}

With multiple decision-makers, as the procedure is expected to finish much quicker if the decision-makers have conflicting objectives, with the time saved, the procedure can be repeated many times, each time with a different initial parameter set $\Omega_{0}$. On the other hand, instead of using a tolerance, a predetermined number should be used as a fixed maximum number of iterations to be carried out in each repeat. Finally, out of all repeats, the solution with the minimum MMR should be used as the final solution. 


\subsection{Hierarchical implementation}

In Defence Force Design, there are primary operational effects (objectives) categories: Land Control, Air Control, Sea Control, Strike etc. Under each of these primary objectives, there are a number of secondary operational effects (objectives) categories: Force Projection and Lift, Sustain the Force, etc. (see Peacock et al. [2019]). One potential approach is to implement RIPE in two phases. In Phase 1, only the primary objectives are used in the RIPE procedures, until the optimal weights $\omega_{1}^{\prime}, \omega_{2}^{\prime}, \omega_{3}^{\prime}, \ldots$ are found. Then in Phase 2 , all secondary objectives are brought in, with the search space of $\Omega$ further constrained by, e.g., the sum of the parameters in the first category must be equal to $\omega_{1}^{\prime}$ and so on.

\section{Conclusions and future work}

In this paper, we presented a framework for Defence Force Design multi-objective decision-making involving multiple decision makers. We proposed some modifications to the Benabbou et al. [2020] method of regretbased incremental preference elicitation. We provided an insight on the impact of having multiple decision makers instead of one decision maker which was originally designed in the Benabbou et al. paper. With multiple decision makers, the search space of the linear scalarizing function parameters in fact converges to a single point much quicker if there are conflicts in terms of preferences among the decision makers. We also proposed a hierarchical approach to deal with very high dimension of the objective space, and proposed alternative approaches to search through the space of scalarizing function parameters. We provide an example in the Appendix, and leave the intended real-life implementation and the tuning of the approach for future research.

\section{ACKNOWLEDGEMENTS}

The research is supported by Collaborative Project Agreement "Scaling Force Effectiveness Modelling and Assessment Methods" between The DSTG and Deakin University.

\section{REFERENCES}

Benabbou, N., Leroy, C. and Lust, T. [2020], 'An interactive regret-based genetic algorithm for solving multiobjective combinatorial optimization problems', Proceedings of the AAAI Conference on Artificial Intelligence 34(03), 2335-2342.

Benabbou, N. and Perny, P. [2015], Incremental weight elicitation for multiobjective state space search, in 'Proceedings of the Twenty-Ninth AAAI Conference on Artificial Intelligence', AAAI'15, AAAI Press, p. 1093-10099.

Benabbou, N. and Perny, P. [2016], Solving multi-agent knapsack problems using incremental approval voting, in 'Proceedings of the Twenty-Second European Conference on Artificial Intelligence', ECAI'16, IOS Press, NLD, p. 1318-1326.

Benabbou, N. and Perny, P. [2018], 'Interactive resolution of multiobjective combinatorial optimization problems by incremental elicitation of criteria weights', EURO Journal on Decision Processes 6, 283-319.

Boutilier, C., Patrascu, R., Poupart, P. and Schuurmans, D. [2006], 'Constraint-based optimization and utility elicitation using the minimax decision criterion', Artif. Intell. 170(8-9), 686-713.

Deb, K., Pratap, A., Agarwal, S. and Meyarivan, T. [2002], 'A fast and elitist multiobjective genetic algorithm:nsga-ii', IEEE Transactions on Evolutionary Computation 6(2).

Hadka, D. [2014], 'MOEA framework user guide: A free and open source java framework for multiobjective optimization (version2.4)', Available at http://www.moeaframework.org/ (accesed in May 2018) .

Hadka, D. and Reed, P. [2012], 'Diagnostic assessment of search controls and failure modes in many-objective evolutionary optimization', Evolutionary Computation 20(3), 423-452.

Kaddani, S., Vanderpooten, D., Vanpeperstraete, J.-M. and Aissi, H. [2017], 'Weighted sum model with partial preference information: Application to multi-objective optimization', European Journal of Operational Research 260(2), 665-679.

Meister, B. and Clauss, P. [2020], Uniform random sampling in polyhedra.

Nguyen, M.-T. and Cao, T. [2017], A hybrid decision making model for evaluating land combat vehicle system, in 'Proc. MODSIM Conference', pp. 1399-1405.

Nguyen, M.-T. and Cao, T. [2019], 'A multi-method approach to evaluate land combat vehicle system', International Journal of Applied Decision Sciences 12(4), 337-360.

Peacock, J., Blumson, D., Mangalasinghe, J., Hepworth, A., Coutts, A. and Lo, E. [2019], Baselining the whole-of-force capability and capacity of the Australian Defence Force, in 'Proc. MODSIM Conference'. 


\section{APPENDIX}

Suppose we have six decision variables $x_{j}$, for $j=1, \ldots, 6$, each with a domain of $\{-1,0,1\}$, and three objective functions $y_{1}(x)=x_{1}+2 x_{2}+2 x_{3}+x_{4}+3 x_{5}+x_{6}, y_{2}(x)=2 x_{1}+4 x_{2}+x_{3}+3 x_{4}+x_{6}$, and $y_{3}(x)=$ $3 x_{1}+x_{2}+2 x_{3}+4 x_{5}+5 x_{6}$. We use a linear scalarizing function $\max z=\omega_{1} y_{1}(x)+\omega_{2} y_{2}(x)+\omega_{3} y_{3}(x)$. The outcomes from Iteration 0 , where four sets of initial scalarizing parameters were used, are presented in the table below. The initial feasible set of the scalarizing parameters is $\Omega_{0}=\left\{\omega_{1}, \omega_{2}, \omega_{2} \geq 0, \omega_{1}+\omega_{2}+\omega_{3}=1\right\}$. After these evaluations in Iteration 0, suppose that a DM, (DM-A), prefers Solution $\left(x^{*}\right)^{0,1}$ over $\left(x^{*}\right)^{0,2}$, we

\begin{tabular}{|c|c|c|c|c|}
\hline \multicolumn{5}{|c|}{ Iteration $0, \Omega_{0}$} \\
\hline & $\omega^{0,1}$ & $\omega^{0,2}$ & $\omega^{0,3}$ & $\omega^{0,4}$ \\
\hline$\omega_{1}^{0, \cdot}$ & 1 & 0 & 0 & 0.4 \\
\hline$\omega_{2}^{0, \cdot}$ & 0 & 1 & 0 & 0.3 \\
\hline$\omega_{3}^{0, \cdot}$ & 0 & 0 & 1 & 0.3 \\
\hline & $\left(x^{*}\right)^{0,1}$ & $\left(x^{*}\right)^{0,2}$ & $\left(x^{*}\right)^{0,3}$ & $\left(x^{*}\right)^{0,4}$ \\
\hline$x_{1}$ & 0 & -1 & 1 & 1 \\
\hline$x_{2}$ & 1 & 1 & 0 & 1 \\
\hline$x_{3}$ & 0 & -1 & 0 & 1 \\
\hline$x_{4}$ & 0 & 1 & -1 & -1 \\
\hline$x_{5}$ & 1 & -1 & 1 & 0 \\
\hline$x_{6}$ & 0 & 1 & 1 & 1 \\
\hline$\overline{f_{\omega^{0}, \cdot}\left(y_{1}(x)\right)}$ & 5 & -2 & 4 & 5 \\
\hline$f_{\omega^{0}, \cdot}\left(y_{2}(x)\right)$ & 4 & 5 & 0 & 5 \\
\hline$f_{\omega^{0}, \cdot}\left(y_{3}(x)\right)$ & 5 & -3 & 12 & 11 \\
\hline
\end{tabular}

Table 1: An example

have that the following cut: $5 \omega_{1}+4 \omega_{2}+5 \omega_{3} \geq-2 \omega_{1}+5 \omega_{2}+-3 \omega_{3}$. Hence,

$$
\Omega_{1}=\left\{\omega_{1}, \omega_{2}, \omega_{2} \geq 0, \omega_{1}+\omega_{2}+\omega_{3}=1,7 \omega_{1}-\omega_{2}+8 \omega_{3} \geq 0\right\}
$$

There may be other preferences from DM-A which will generate more cuts. If, however, there is another DM, (DM-B), whose preference is the opposite, the cut will be $7 \omega_{1}-\omega_{2}+8 \omega_{3} \leq 0$, The contradicting preferences will give us the equation $7 \omega_{1}-\omega_{2}+8 \omega_{3}=0$ and as soon as we have a third equation that is linearly independent to $\omega_{1}+\omega_{2}+\omega_{3}=1$ and $7 \omega_{1}-\omega_{2}+8 \omega_{3}=0$, we will have a unique solution for $\omega_{1}, \omega_{2}, \omega_{3}$. Then, one can solve a SOO with these parameter values to obtain the optimal values of the original decision variables $x_{1}, \ldots, x_{6}$. 